TP Periodica Polytechnica Electrical Engineering and Computer Science

61(1), pp. 77-81, 2017

DOI: 10.3311/PPee.10379

Creative Commons Attribution (i)

RESEARCH ARTICLE

\section{Neutral Voltage Comparison of Different Grounding Configurations and Calculation Methods in Multi- Grounded Low Voltage Network}

\author{
János Csatár ${ }^{1 *}$, András Dán ${ }^{1}$
}

Received 09 December 2016; accepted after revision 06 January 2017

\begin{abstract}
The steady growth in distributed generation brings more and more attention to low voltage (LV) distribution networks. Therefore, a good model is important to study these trends, especially with local regulation. Grounding the neutral line at multiple points along the network is a common practice in many European countries influencing neutral currents and voltages. Modelling grounding current had been of little interest at network planning and operation before, with only passive consumers. However, with the growing number of smart equipment, it becomes an overlooked, yet important issue. This equipment usually rely on local measurements and regulates accordingly. Given that most of the LV equipment uses singlephase connection, the neutral voltage is an important issue. Yet few papers study the neutral line behaviour and often overlook the effect of grounding. This article studies the effect of different grounding configurations on neutral voltages, and compares it with one of the most commonly used calculation method in literature that incorporates groundings. The differences are large enough to adversely affect local regulation.
\end{abstract}

\section{Keywords}

low voltage network, grounding, unbalance, neutral voltage

\section{Introduction}

With the steady growth of distributed generation, more and more study deal with the effects on low voltage (LV) grids. Several works neglected neutral to earth voltage and its effect on the single phase connected equipment, or even assumed balanced networks (e.g. [1-5]). These studies all used well-developed methods; however, the effect of unbalance and neutral line could be substantial, as shown in this article.

Many European low voltage networks are of multi-grounded four-wire type: three phases and one neutral. This neutral line is grounded at multiple points along the network. Single-phase consumers and generating units are connected between phase and neutral. Thus, the correct modelling of neutral voltage and current distribution between neutral and ground plays an important role. In [6] the authors, although for medium voltage (MV) network, used EMTP software to show that voltage drop on the phase conductor can well be in the same order than the neutral voltage, [6] also presented the unique $U$ shape of the neutral voltage profile. Reference [7] clearly showed the effect through a small example. More precise modelling requires more sophisticated calculation tools and new challenges (e.g. [8]).

Not only is the magnitude of the neutral voltage important but also the angle, because load and generation on other phases also connect to this common neutral. Even more, common three-phase inverters also measure phase to neutral voltages for regulation. Therefore, neutral line and its grounding worth a closer look. This article deals with the effect of grounding on low voltage networks. The point is to highlight these through a simple, yet realistic example. Another aim is to compare the more precise results with two common practices in studies: neglecting groundings and only calculating ground current injections at load nodes with ideal transformer grounding.

\section{Multi-grounded networks and a simple model}

Many European countries have widespread, geographically extensive low voltage networks. In these cases, the total length of the LV network is generally larger than that of the MV network. As such, the LV networks are longer, compared to the usual North-American type networks. Because of that,

\footnotetext{
${ }^{1}$ Department of Electric Power Engineering, Faculty of Electrical Engineering and Informatics, Budapest University of Technology and Economics H-1521 Budapest, P.O.B. 91, Hungary

*Corresponding author, e-mail: csatar.janos@vet.bme.hu
} 
grounding at multiple points along the line is important. There can be much variation in load and its distribution among phases due to the relatively small number of consumers.

As an example from Hungary ([9]) [10]: The average overhead line length (the farthest point from the transformer) is around $750 \mathrm{~m}$, with most of the lines being between $250 \mathrm{~m}$ and $1500 \mathrm{~m}$. Overhead lines generally have 1 to 4 laterals. Usually three consumers connect to the network at every pole (around every $30 \mathrm{~m}$ ) in suburban areas.

The model network is a medium-long LV feeder; these hold a large share in the network. Laterals are not modelled here for the sake of simplicity. The overall loads of the phases are close to each other. However, the unbalance at a node level is not necessarily small, in fact quite the opposite because consumption behaviour is different from household to household; this is still one of the main assumptions at network planning.

Summing the above, the base model network (Fig. 1 and Fig. 2) has the following attributes:

- A single line.

- $960 \mathrm{~m}$ total length

- $95 \mathrm{~mm}^{2}$ bare wire overhead line with aluminium conductors in lateral configuration

- Grounding at every $30 \mathrm{~m}$ (pole distance).

- $1 \Omega$ at the transformer - transformer neutral grounding and other line's equivalent grounding resistance

- $30 \Omega$ at every pole except the end, where it is $10 \Omega$

- Consumers:

- Three consumers at every pole $(30 \mathrm{~m})$, connected to different phases

- Constant power type

- Connected between phase and neutral

- Have a random power factor between 0.98 and 0.999

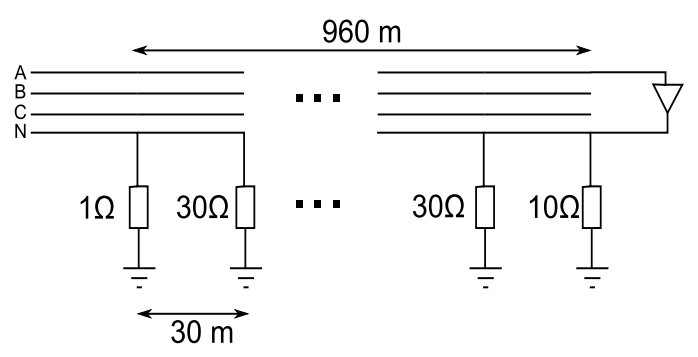

Fig. 1 The basic layout of the model network.

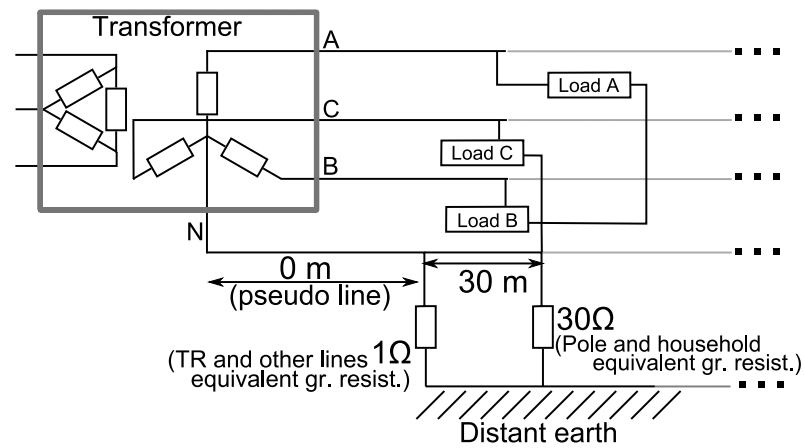

These grounding resistances may seem large at a first glance, but they represent the true grounding resistance to the ideal earth, not to local earth (e.g. [10]). It is important that the transformer grounding is not ideal.

The average of the active loads per phase per connection point on the network is $600 \mathrm{~W}$; the maximum is $3000 \mathrm{~W}$. This corresponds with a moderately loaded network, nor at peak nor at valley period.

Table 1 Loads at nodes - active power

\begin{tabular}{|c|c|c|c|}
\hline Distance (m) & $\begin{array}{l}\text { Phase A } \\
\text { load(W) }\end{array}$ & $\begin{array}{l}\text { Phase B } \\
\operatorname{load}(W)\end{array}$ & $\begin{array}{l}\text { Phase C } \\
\operatorname{load}(W)\end{array}$ \\
\hline 0 & 0 & 0 & 900 \\
\hline 30 & 100 & 1500 & 1800 \\
\hline 60 & 300 & 400 & 0 \\
\hline 90 & 1800 & 100 & 200 \\
\hline 120 & 900 & 200 & 300 \\
\hline 150 & 200 & 300 & 300 \\
\hline 180 & 400 & 100 & 400 \\
\hline 210 & 100 & 50 & 400 \\
\hline 240 & 2000 & 2000 & 600 \\
\hline 270 & 1700 & 300 & 500 \\
\hline 300 & 300 & 80 & 2000 \\
\hline 330 & 600 & 0 & 100 \\
\hline 360 & 710 & 900 & 200 \\
\hline 390 & 100 & 2000 & 200 \\
\hline 420 & 400 & 400 & 400 \\
\hline 450 & 200 & 50 & 100 \\
\hline 480 & 3000 & 400 & 600 \\
\hline 510 & 200 & 100 & 300 \\
\hline 540 & 80 & 150 & 200 \\
\hline 570 & 150 & 1500 & 0 \\
\hline 600 & 600 & 300 & 400 \\
\hline 630 & 400 & 100 & 600 \\
\hline 660 & 300 & 200 & 1500 \\
\hline 690 & 2000 & 600 & 2000 \\
\hline 720 & 300 & 500 & 300 \\
\hline 750 & 500 & 0 & 0 \\
\hline 780 & 800 & 2000 & 200 \\
\hline 810 & 50 & 200 & 1500 \\
\hline 840 & 600 & 1500 & 300 \\
\hline 870 & 100 & 800 & 400 \\
\hline 900 & 200 & 200 & 300 \\
\hline 930 & 400 & 3000 & 400 \\
\hline 960 & 1500 & 200 & 400 \\
\hline Total & 20990 & 20130 & 17800 \\
\hline
\end{tabular}

Fig. 2 Detailed view of the start of the model network. 


\section{Calculation results}

The focus is on neutral voltage change, thus the ground voltage rise near the individual groundings was not calculated. Two method was used; the main difference lies in how the current through grounding is calculated:

- Method A: a popular one among load-flow studies that distributes current among neutral and ground based only on network impedances at local injection alone:

$$
I_{g r}=\frac{Z_{g}}{Z_{g}+Z_{n w}}\left(I_{a}+I_{b}+I_{c}\right)
$$

where $I_{a}, I_{b}, I_{c}$ denotes the load currents in phases A, $\mathrm{B}$ and $\mathrm{C}$ respectively, $Z_{g}$ denotes the system equivalent impedance through grounding, $Z_{n w}$ denotes the system equivalent impedance in neutral and $I_{g r}$ denotes the current through grounding; it is similar to [11]

- Method B: A more precise way, with a software based on [12] and [13], considering neutral voltages and grounding resistances, currents.

The simulations used the following cases:

- Case A - Base:

the base network and load condition using method B

- Case B - Isolated Neutral:

same as case A except the grounding resistances are changed to $9999 \Omega$ only the transformer grounding is 0 $\Omega$ - basically isolated neutral on the network (neglecting grounding resistance)

- Case C-Reduced Network Grounding Resistance: the same as case A except the reduced grounding resistances at consumer connection points: from $30 \Omega$ to $10 \Omega$ and from $10 \Omega$ to $5 \Omega$

- Case D - Increased Network Grounding Resistance: the same as case A except the increased grounding resistances at consumer connection points: from $30 \Omega$ to $60 \Omega$ and from $10 \Omega$ to $20 \Omega$

- Case E - Increased Transformer Grounding Resistance: the same as case A except the increased transformer grounding resistance: from $1 \Omega$ to $2 \Omega$

- Case F - Reduced Transformer Grounding Resistance: the same as case A except the reduced transformer grounding resistance: from $1 \Omega$ to $0.5 \Omega$

- Case G-Base with Method A:

the same as case A except and that it uses method A therefore, the transformer grounding resistance is $0 \Omega$

These cases show the effect of changing grounding resistance and the effect of simplification in calculation method.

Fig. 3 and Fig. 4 show the calculated neutral voltages of the cases. The following observations are conspicuous:

- Using method A results in almost no change from the isolated neutral case.
- With constant transformer grounding resistance, the smaller the resistances of groundings along the line

- the smaller the neutral voltage amplitude towards the end of the line.

- the larger the neutral voltage amplitude at the start of the line.

- With constant grounding resistances along the line, the smaller the transformer grounding resistance

- the larger the neutral voltage amplitude towards the end of the line.

- the smaller the neutral voltage amplitude at the start of the line.

- The neutral voltage amplitude is not necessarily smaller closer to the transformer.

- The calculation method completely changes the neutral voltage profile.

The neutral voltage profiles of case B and G (Isolated Neutral and Base case with method A) show that:

- The neutral voltage effect on the phase-to-neutral voltages changes close to linearly with distance.

- And the neutral voltage change consist of mainly amplitude change, thus affecting the phases to the same proportion throughout the network.

In contrast, the neutral voltage profiles in the rest of the cases (A, C, D, E and F) show that:

- The neutral voltage effect on the phase-to-neutral voltages changes

- with distance

- not only in amplitude but also in which phases it has the most impact on

- And the neutral voltage effect proportion on the phases varies throughout the network.

Therefore, neglecting grounding path or incorrectly defining currents through grounding changes the neutral behaviour of the network.

What truly defines the shape of the neutral voltage profile is the relative grounding resistances to each other. Moreover the smaller the resistances, the smaller the neutral voltage amplitude. Generally, voltage amplitude is larger towards the end and towards the start of the network. The angle of the neutral voltage is constantly changing; it goes through an almost 180 degrees turn. Despite the relatively large neutral voltage at the start of the feeder, the effect on phase to neutral voltage here is small. This is due to neutral point shift on the transformer where the phase to neutral voltages remain the same. 


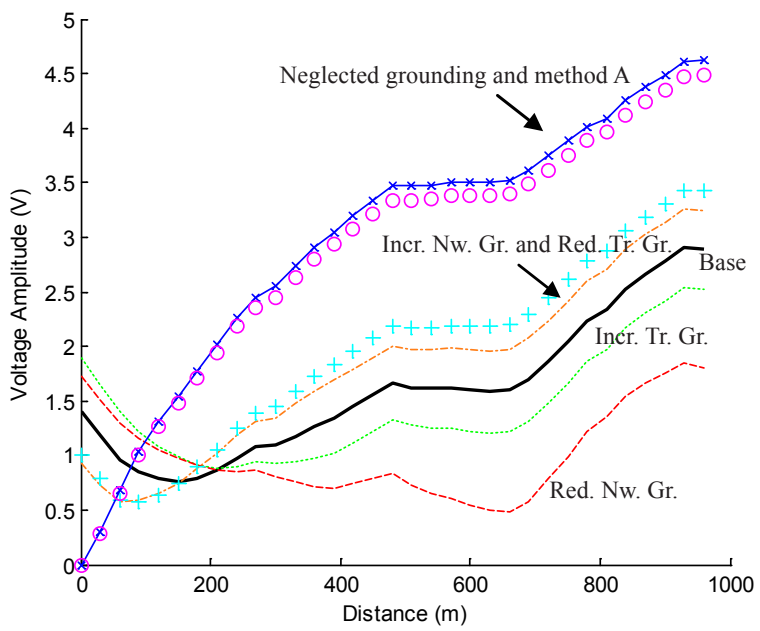

Fig. 3 Neutral voltage amplitudes. Black - Case A (Base), Blue with cross Case B (Isolated Neutral), Red-dashed - Case C (Red. Nw. Gr.), Cyan crosses - Case D (Incr. Nw. Gr.), Green-dotted - Case E (Incr. Tr. Gr.), Orange-dotdashed - Case F (Red. Tr. Gr.), Magenta circles- Case G (Base with Method A)

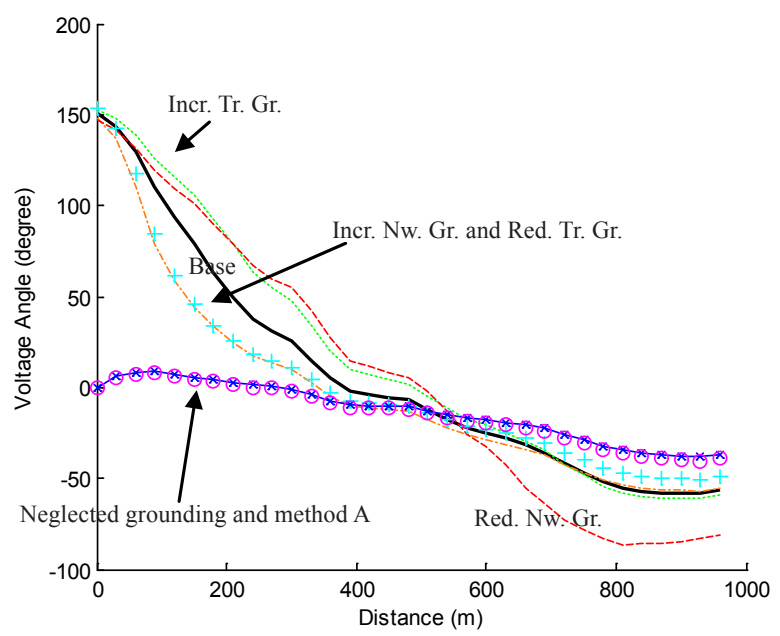

Fig. 4 Neutral voltage angles. Black - Case A (Base), Blue with cross - Case B (Isolated Neutral), Red-dashed - Case C (Red. Nw. Gr.), Cyan crosses - Case $D$ (Incr. Nw. Gr.), Green-dotted - Case E (Incr. Tr. Gr.), Orange-dot-dashed Case F (Red. Tr. Gr.), Magenta circles- Case G (Base with Method A)

While the cases show distinctive voltage profile, the current amplitude and angle change in the neutral line is small between cases as Fig. 5 and Fig. 6 show it. The amplitude change is due to different loading at every $30 \mathrm{~m}$, where consumers connect to network.

Losses for the whole system are about the same, they do not differ from each other more than $0.2 \%$.

Changing various characteristics of the network has the following general effect on neutral voltages:

- Number of laterals: This adds more possible connection points to the network, without increasing the maximal distance of the consumers. Alone by itself it has minimal impact but brings more possible load state and possible

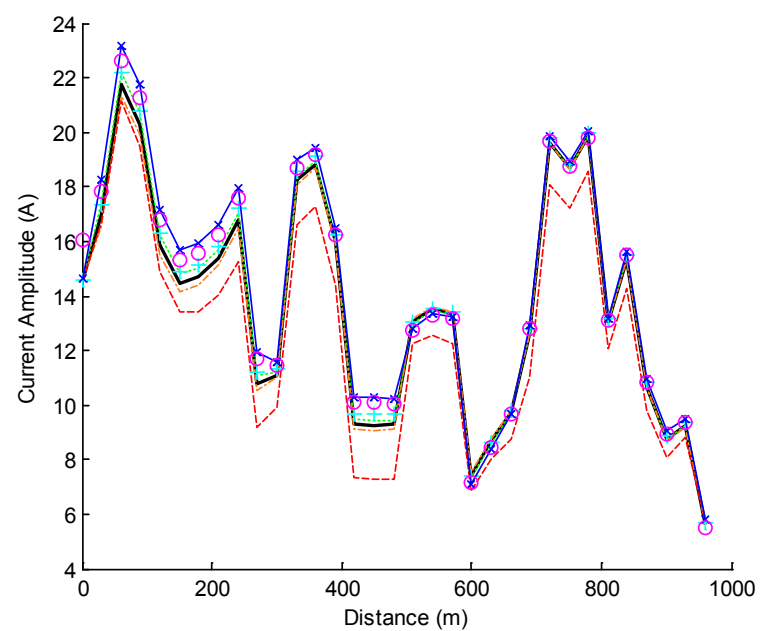

Fig. 5 Neutral current amplitudes. Black - Case A (Base), Blue with cross Case B (Isolated Neutral), Red-dashed - Case C (Red. Nw. Gr.), Cyan crosses - Case D (Incr. Nw. Gr.), Green-dotted - Case E (Incr. Tr. Gr.), Orange-dotdashed - Case F (Red. Tr. Gr.), Magenta circles- Case G (Base with Method A)

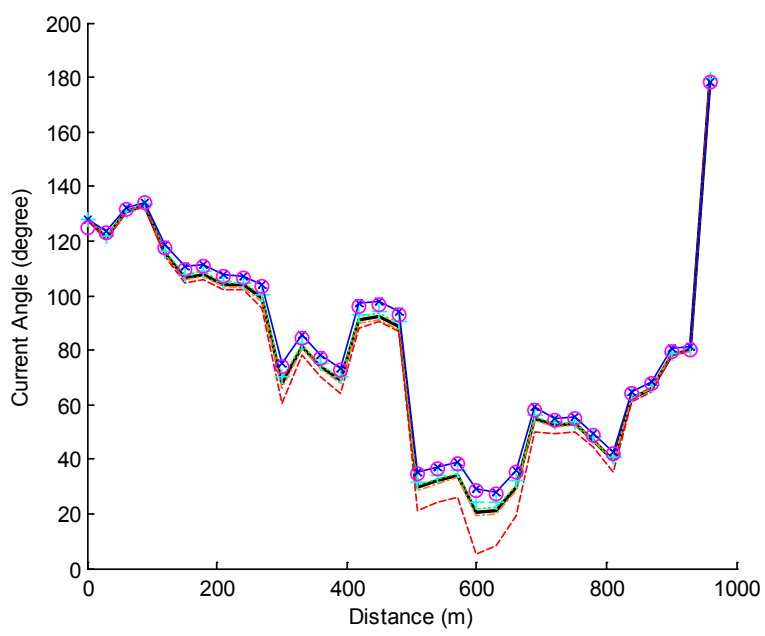

Fig. 6 Neutral current angles. Black - Case A (Base), Blue with cross - Case B (Isolated Neutral), Red-dashed-Case C (Red. Nw. Gr.), Cyan crosses-Case $D$ (Incr. Nw. Gr.), Green-dotted - Case E (Incr. Tr. Gr.), Orange-dot-dashedCase F (Red. Tr. Gr.), Magenta circles- Case G (Base with Method A)

grounding configurations to the network, which could heavily affect neutral line.

- Length of lines: The longer the line, the more possible grounding points on the network can appear. This brings the neutral voltage closer to ideal earth at the middle of the line. Longer lines also means more line impedance, thus larger neutral voltages can appear.

- Grounding resistances: The greater the resistances the less current goes through ground. Surprisingly, even relatively large individual groundings still has a significant impact on neutral voltage.

- Load unbalance: The more unbalanced the network is the more neutral current flows in the neutral line. Therefore, the neutral voltage amplitude grows. 
Nevertheless, the general shape of the profile and the constantly changing angle along the line is apparent in all cases; except with isolated neutral and calculating with method A.

Therefore, all simulations using a calculation method that is similar to method A or neglecting the grounding resistances are inadequate for neutral-earth voltage modelling with multi grounded networks incorporating unbalanced loads. The neutral voltage profile is also different with ideal transformer grounding from the usual ' $U$ ' shaped profiles (e.g. based on test system described in [14]).

\section{Conclusion}

The calculations show that asymmetrical voltages could appear even though the system appears almost balanced judged from phase current measurements at the start of the feeder. The phases in the model network are similarly loaded on a feeder level, but not on a consumer level; this stems from households' consumption behaviour. Moreover, having different load in phases at feeder level is not uncommon on LV networks; this would increase the unbalance also on a node level.

For the test network, the currents in the neutral line barely change with different grounding configurations and calculation methods (as seen on Fig. 5 and Fig. 6). However, these do affect currents through grounding and the neutral voltage profile (as seen Fig. 3 and Fig. 4). The loss change in the network is only marginal due to small changes in currents and resistance in the ground path.

The neutral voltage becomes a few volts in every case, but the amplitude and angle is entirely different if one takes into account the ground path correctly i.e. neglecting grounding or using method A is inappropriate. The effect of neutral line on phase-to-neutral voltages also varies with different configurations and calculation methods (as seen Fig. 3 and Fig. 4). The differences are at a scale where it can offset anticipated local regulation behaviour. This is important for smart grid equipment, where local regulation is present also on low voltage networks.

Although the model network does not represent all possible network topologies, the basic principles should hold true to all four-wire, multi-grounded networks: the general shape of neutral voltage profile, the constantly changing neutral voltage angle, effect of different grounding configuration on neutral voltage.

\section{References}

[1] Supponen, A., Repo, S. "Impact evaluation of PV generation on LV networks." In: Power Systems Computation Conference (PSCC), 2014, Wroclaw, Poland, Aug. 18-22, 2014. https://doi.org/10.1109/pscc.2014.7038331

[2] García, A. M., Mastromauro, R. A., Liserre, M. "A combined centralized/ decentralized voltage regulation method for PV inverters in LV distribution networks." In: 2014 IEEE PES General Meeting | Conference \& Exposition, National Harbor, MD, July 27-31, 2014, pp. 1-5. https://doi.org/10.1109/PESGM.2014.6938817
[3] Wang, Z., Qin, L., Gu, C., Li, F. "Distributed storage capacity reservations for residential PV generation utilization and LV network operation." In: 2015 IEEE Power \& Energy Society General Meeting, Denver, CO, July 26-30, 2015, pp. 1-5.

https://doi.org/10.1109/PESGM.2015.7286114

[4] Kabiri, R., Holmes, D. G., McGrath, B. P. "Voltage regulation of LV feeders with high penetration of PV distributed generation using electronic tap changing transformers." In: Power Engineering Conference (AUPEC), 2014 Australasian Universities, Perth, WA, 28 Sept.-1 Oct. 2014, pp. 1-6. https://doi.org/10.1109/AUPEC.2014.6966635

[5] Wang, Y., Tan, K. T., Peng, X. Y., So, P. L. "Coordinated Control of Distributed Energy-Storage Systems for Voltage Regulation in Distribution Networks." IEEE Transactions on Power Delivery. 31(3), pp. 1132-1141. 2016 https://doi.org/10.1109/TPWRD.2015.2462723

[6] Chen, T.-H., Yang, W.-C. "Analysis of Multi-Grounded Four-Wire Distribution Systems Considering the Neutral Grounding." IEEE Transactions on Power Delivery. 16(4), pp. 710-717. 2001.

https://doi.org/10.1109/61.956760

[7] Carvalho, P. M. S., Ferreira, L. A. F. M., Santana, J. J. E. "Single-Phase Generation Headroom in Low-Voltage Distribution Networks Under Reduced Circuit Characterization." IEEE Transactions on Power Systems. 30(2), pp. 1006-1011. 2015. https://doi.org/10.1109/TPWRS.2014.2337712

[8] de Araujo, L. R., Penido, D. R. R., Carneiro, S., Pereira, J. L. R. "A Study of Neutral Conductors and Grounding Impacts on the Load-Flow Solutions of Unbalanced Distribution Systems." IEEE Transactions on Power Systems. 31(5), pp. 3684-3692. 2016. https://doi.org/10.1109/TPWRS.2015.2494380

[9] Csatár, J., Dán, A. "LV Grid Modeling Including Consumption and Distributed PV Generation with Focus on Voltage Profile." Periodica Polytechnica Electrical Engineering and Computer Science. 59(3), pp. 110-117. 2015. https://doi.org/10.3311/PPee.8574

[10] Papathanassiou, S., Hatziargyriou, N., Strunz, K. "A Benchmark Low Voltage Microgrid Network." In: CIGRE Symposium Power systems with dispersed generation: Technologies, impacts on development, operation and performances, April 2005.

[11] Ciric, R. M., Feltrin, A. P., Ochoa, L. F. "Power flow in four-wire distribution networks-general approach." IEEE Transactions on Power Systems. 18(4), pp. 1283-1290. 2003.

https://doi.org/10.1109/TPWRS.2003.818597

[12] Károlyi, K., Varjú, Gy. "Multipoint boundary value problems for calculating the screening effect of a metal cable sheath with nonlinearitiy due to steel armouring." Annales Universitatis Scientiarum Budapestinensis de Rolando Eotvos Nominatae Sectio Computatorica. 10, pp. 109-134. 1989.

[13] Sollerkvist, F. J., Varjú, G. "A General Model and Numerical Method for Multiconductor Systems in the Frequency Domain." In: IEEE/KTH Power Tech. Conf., Stockholm, Sweden, June 18-22, 1995, Paper SPT HV 07-08-0302.

[14] Sunderman, W. G., Dugan, R. C., Dorr, D. S. "The neutral-to-earth voltage (NEV) test case and distribution system analysis." In: 2008 IEEE Power and Energy Society General Meeting - Conversion and Delivery of Electrical Energy in the 21st Century, Pittsburgh, PA, July 20-24, 2008, pp. 1-6. https://doi.org/10.1109/PES.2008.4596812 